\title{
Mandibular tori
}

\section{Maxime Mermod MD, Remy Hoarau MD}

\section{Competing interests:} None declared.

This article has been peer reviewed.

The authors have obtained patient consent.

\section{Affiliations: Service} d'oto-rhino-laryngologie et chirurgie cervico-faciale (Mermod) and Unité de chirurgie maxillo-faciale (Hoarau), Centre hospitalier universitaire Vaudois, Lausanne, Switzerland

Correspondence to: .mermod@gmail.com

CMAJ 2015. DOI:10.1503 /cmaj.141048

A Maxime Mermod, maxime 44-year-old man was referred for treatment of symptomatic sialolithiasis in the right Wharton duct. Intraoral removal could not be performed because of bilateral swellings on the lingual surface of the mandible. The patient was unable to recall when he first became aware of the lesions. He had no history of mandibular trauma or surgery. Physical examination showed two symmetric, nontender, bony outgrowths on the lingual surface of the mandible (Figure 1). We diagnosed mandibular tori. Although the lesions were causing only minor symptoms, we elected to remove them to gain access to the Wharton duct.

Torus mandibularis is a nontender, bony outgrowth located on the lingual side of the mandible, in the canine or premolar region, above the attachment of the mylohyoid muscle. In most cases, bilateral tori are present. ${ }^{1}$ Torus mandibularis is usually asymptomatic and discovered incidentally. The prevalence varies substantially between ethnic groups, with lower prevalence in

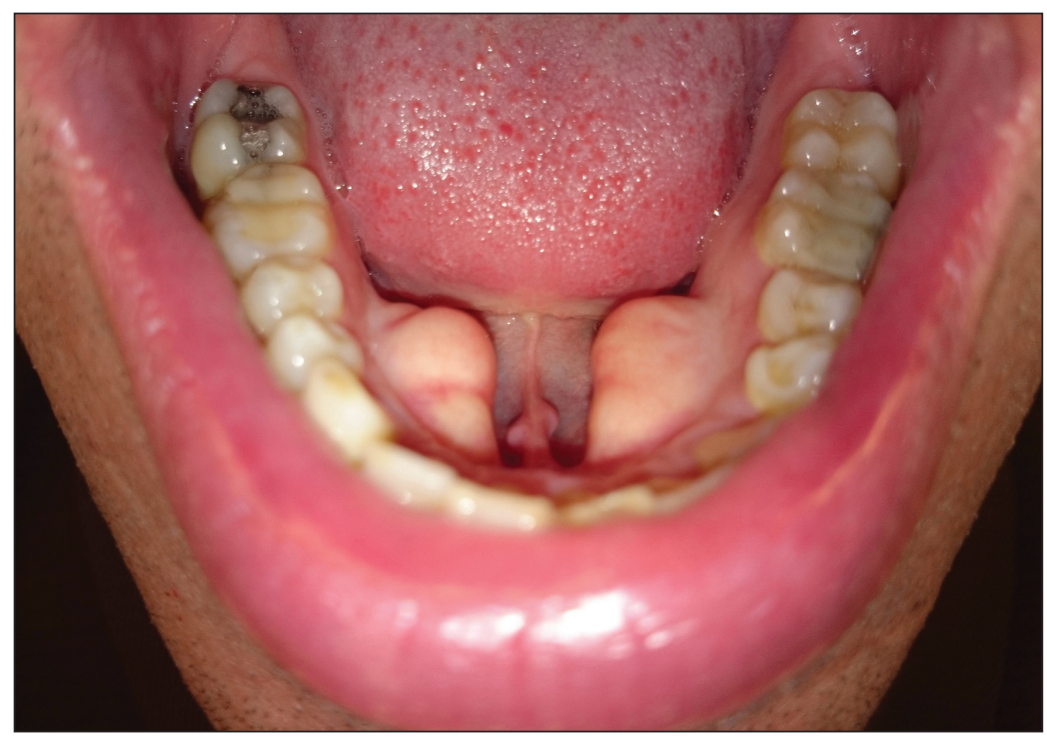

Figure 1: Mandibular tori are bony outgrowths on the lingual surface of the mandible, often bilateral and symmetric, as in this 44-year-old patient. whites (about 8\%) and blacks (about 16\%) and higher prevalence in Asian and Inuit populations. ${ }^{2}$ Torus mandibularis is slightly more common in males than in females. ${ }^{3}$ Histologic examination shows dense bony tissue, with normal osteocytes and lacunae. ${ }^{3}$

Torus mandibularis is thought to be caused mainly by environmental factors, such as bruxism, vitamin deficiencies and calcium-rich supplements, although genetic background also plays a key role. ${ }^{1}$ Clinical diagnosis is usually straightforward, and investigations are generally not required. However, peripheral ossifying fibroma, osteoma, osteochondroma, osteoid osteoma, osteoblastoma and osteosarcoma should also be considered in the differential diagnosis of a unilateral, growing lesion. ${ }^{4}$ In particular, the presence of pain or paresthesia should prompt further investigation.

The growth of torus mandibularis is very slow and may stop spontaneously. ${ }^{1}$ Surgical resection is seldom necessary, but is indicated when ulceration, articulation disorder or problems inserting dentures are present. ${ }^{3}$

\section{References}

1. García-García A, Martinez-Gonzalez J, Gomez-Font R, et al. Current status of the torus palatinus and torus mandibularis. Med Oral Patol Oral Cir Bucal 2010;15:e353-60.

2. Patil S, Maheshwari S, Khandelwal S. Prevalence of torus palatinus and torus mandibularis in an Indian population. Saudi J Oral Sci 2014;1:94-7.

3. Loukas M, Hulsberg P, Tubbs RS, et al. The tori of the mouth and ear: a review. Clin Anat 2013;26:953-60.

4. Sayan NB, Ucok C, Karasu HA, et al. Peripheral osteoma of the oral and maxillofacial region: a study of 35 new cases. J Oral Maxillofac Surg 2002;60:1299-301.

Clinical images are chosen because they are particularly intriguing, classic or dramatic. Submissions of clear, appropriately labelled high-resolution images must be accompanied by a figure caption and the patient's written consent for publication. A brief explanation (250 words maximum) of the educational significance of the images with minimal references is required. 